\title{
Effect of Fracture Aperture on P-Wave Attenuation: A Seismic Physical Modelling Study
}

\author{
Aniekan Martin Ekanem, ${ }^{1,2,3}$ Xiang Yang Li, ${ }^{3,4}$ \\ Mark Chapman, ${ }^{2,3}$ Main Ian, ${ }^{2}$ and Jianxin Wei ${ }^{4}$ \\ ${ }^{1}$ Department of Physics, Akwa Ibom State University, PMB 1167, Mkpat Enin, Nigeria \\ ${ }^{2}$ School of Geosciences, University of Edinburgh, Edinburgh EH9 3JW, UK \\ ${ }^{3}$ British Geological Survey, Murchison House, Edinburgh EH9 3LA, UK \\ ${ }^{4}$ CNPC Geophysical Key Laboratory, China University of Petroleum, Beijing 102249, China
}

Correspondence should be addressed to Aniekan Martin Ekanem; anny4mart@yahoo.com

Received 14 August 2013; Accepted 24 December 2013; Published 11 February 2014

Academic Editors: E. Del Pezzo, E. Liu, F. Luzon Martinez, A. Stovas, and T. Tsapanos

Copyright (C) 2014 Aniekan Martin Ekanem et al. This is an open access article distributed under the Creative Commons Attribution License, which permits unrestricted use, distribution, and reproduction in any medium, provided the original work is properly cited.

\begin{abstract}
We used the seismic physical modelling approach to study the effect of fracture thickness or aperture on P-wave attenuation, using a laboratory scale model of two horizontal layers. The first layer is isotropic while the second layer has six fractured blocks, each consisting of thin penny-shaped chips of $3 \mathrm{~mm}$ fixed diameter and same thickness to simulate a set of aligned vertical fractures. The thickness of the chips varies according to the blocks while the fracture density remains the same in each block. 2D reflection data were acquired with the physical model submerged in a water tank in a direction perpendicular to the fracture strikes using the pulse and transmission method. The induced attenuation was estimated from the preprocessed CMP gathers using the QVO method, which is an extension of the classical spectral ratio method of attenuation measurement from seismic data. The results of our analysis show a direct relationship between attenuation and the fracture thickness or aperture. The induced attenuation increases systematically with fracture thickness, implying more scattering of the wave energy in the direction of increasing aperture. This information may be useful to differentiate the effect caused by thin microcracks from that of large open fractures.
\end{abstract}

\section{Introduction}

Fractures open at depth tend to be aligned normal to the direction of minimum in situ stress acting on them [1], giving rise to seismic anisotropy. Over the years, seismic anisotropy has been increasingly used as a potential tool to characterize natural fractured hydrocarbon reservoirs (e.g., [2-5]). The equivalent medium theories of seismic wave propagation provide the basis of using seismic anisotropy to detect fractures from seismic data. One of such theories is the Hudson's theory $[6,7]$ which provides a link between fracture density and measured azimuthal anisotropy. However, the theory fails to account for the issues of fracture scale lengths. For instance, many small cracks or a few large cracks within the same volume of material can result in the same fracture density. Furthermore, equal number of cracks with the same diameter but with varying thicknesses or apertures within the same volume of material can give rise to the same fracture density.
In reservoir rocks, it is possible to have aligned fractures of the same density but at different scales and consequently, the investigation of the effects of the fracture scale lengths and thicknesses or apertures on seismic wave response may be of great interest in fractured reservoir characterization. An adequate understanding of these effects could provide useful information needed to differentiate between the effects caused by thin microcracks and large open fractures. This is particularly important because key engineering quantities such as fluid transmissivity, used in reservoir models, depend on the third power of the hydraulic aperture and even more strongly on a hydraulic aperture below a few microns, where a significant proportion of the two opposing rough fractures are in contact [8].

Wei et al. $[9,10]$ used the seismic physical modelling approach to examine the effects of fracture scale lengths on seismic wave velocity and amplitude for both P- and Stransmitted waves. They simulated a set of aligned fractures 


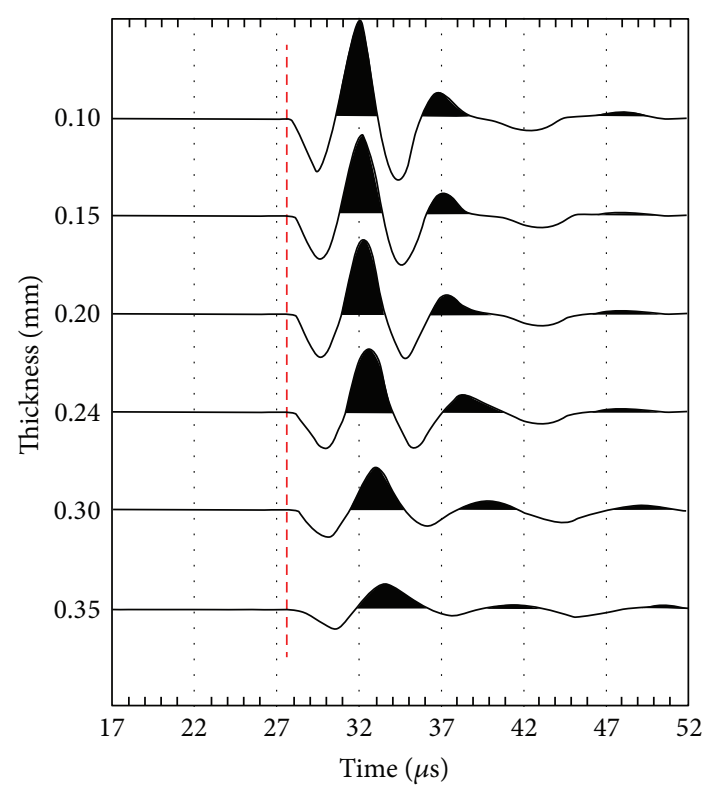

(a)

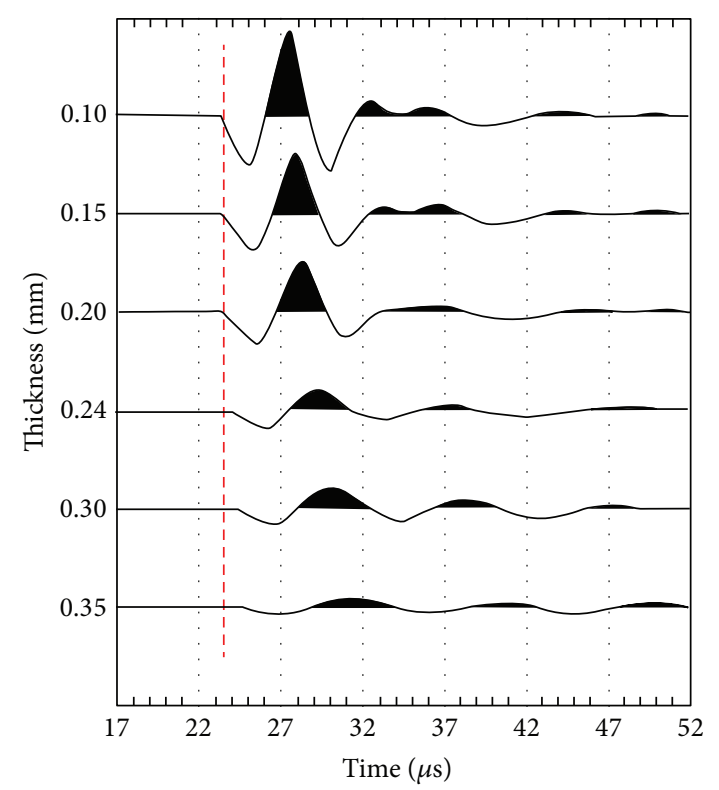

(b)

Figure 1: Comparison of P-waves recorded for the fractured models with different thicknesses or apertures (from [9]). (a) Parallel to the fracture strike. (b) Perpendicular to fracture strike. The wave is attenuated more in the direction perpendicular to fractures.

by embedding thin penny-shaped low-velocity chips of the same density and thickness $(0.14 \mathrm{~mm})$ but varying diameter (ranging from $2.5 \mathrm{~mm}$ to $6.0 \mathrm{~mm}$ ) into an isotropic background material on the basis of Hudson's [6] assumption of thin penny-shaped fractures and used the pulse and transmission method to study the influence of the fracture diameter on the transmitted data. The results of their studies show that both $\mathrm{P}$ - and $\mathrm{S}$-wave velocities increase with fracture diameter especially for wave propagation parallel to the fracture strike. They argued that as the diameter increases the number of fractures decreases to keep the density constant, resulting in a decrease in the amount of scattering and an increase in the wave velocity. Their results also reveal that the amount of shear-wave splitting decreases as the fracture diameter increases also as a result of the reduction in the number of fractures as the diameter increases. Wei et al. [9] further investigated the influence of fracture thickness or aperture on the P-waves by embedding thin round chips of the same diameter $(2.1 \mathrm{~mm})$ but varying thicknesses (ranging from $0.1 \mathrm{~mm}$ to $0.35 \mathrm{~mm}$ ) into an isotropic background material to simulate a set of aligned fractures with different apertures. Their results show significant changes in the Pwave amplitude and waveforms with increasing thicknesses or apertures. As the thickness of the chips increases, the Pwave is significantly attenuated as illustrated in Figure 1 with more attenuation perpendicular to the fractures. However, their study fails to provide quantitative estimates of the observed attenuation from the waveforms. Chapman [11] developed a poroelastic model to account for the effects of fracture scale lengths, but there is still a lack of adequate understanding of the effects of fracture thickness or aperture on seismic P-wave attenuation. In this paper, we extend the previous studies by Wei et al. [9] to seismic reflection data by estimating the amount of scattered attenuation caused by fractures of varying thicknesses or apertures but same density to provide more understanding of the scattering effects on $\mathrm{P}$ wave amplitude.

The modelling involves first building the physical scalemodel in the laboratory and then using the pulse and transmission method to record the seismic reflection response in the model. The resulting data though acquired in the laboratory have similar features as the data acquired in the field, and hence the results of the attenuation analysis could provide valuable information to differentiate between the effects caused by thin microcracks and large open fractures from field data. The setup of the seismic physical modelling experiment is inspired by Hudson's equivalent medium theory $[6,7]$ which considers dilute inclusions of thin, penny-shaped ellipsoidal cracks in an isotropic background medium. A known number of round thin low-velocity chips with fixed diameter but varying thicknesses or apertures are embedded into an isotropic base material to simulate a set of aligned vertical fractures with varying apertures and the fracture density is derived based on Hudson's theory [6, 7].

\section{Construction of the Physical Model}

The physical model is constructed from two horizontal layers (Figure 2). The first layer is made from a mixture of epoxy resin and silicon rubber and has a thickness of $38 \mathrm{~mm}$, P-wave velocity of $2150 \mathrm{~m} / \mathrm{s}$, S-wave velocity of $1100 \mathrm{~m} / \mathrm{s}$, and density of $1.15 \mathrm{~g} / \mathrm{cm}^{3}$. The second layer is made from epoxy resin with a thickness of $75.5 \mathrm{~mm}$, P-wave velocity of $2573 \mathrm{~m} / \mathrm{s}$, S-wave velocity of $1200 \mathrm{~m} / \mathrm{s}$, and density of $1.18 \mathrm{~g} / \mathrm{cm}^{3}$. To simulate fractures with varying apertures, thin penny-shaped chips made from a mixture of epoxy resin and silicon rubber with 


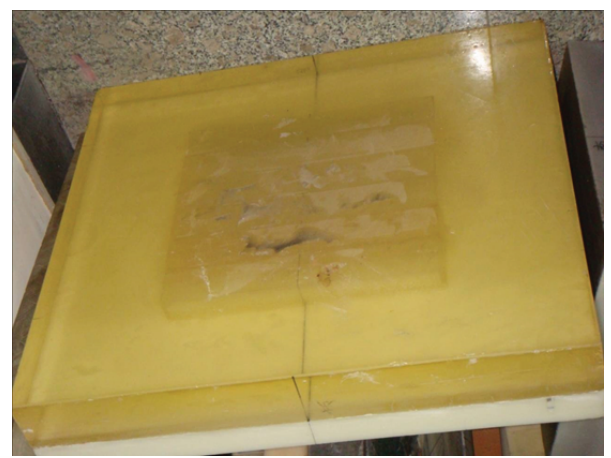

(a)

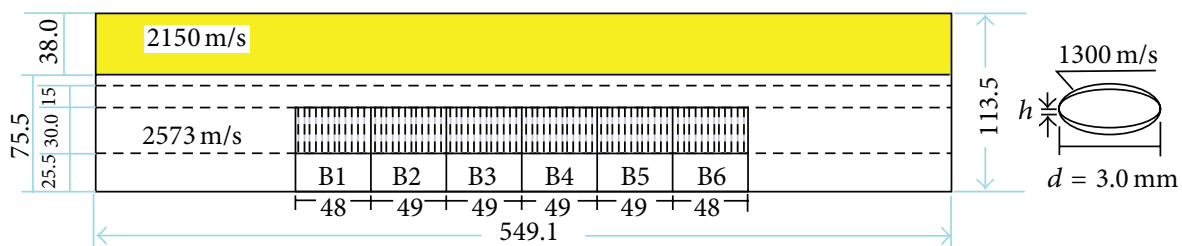

(b)

Figure 2: Physical model made up of two layers: (a) base model, (b) sectional view of base model. The first layer is isotropic with a Pwave velocity of $2150 \mathrm{~m} / \mathrm{s}$ and density of $1.15 \mathrm{~g} / \mathrm{cm}^{3}$ while the second layer has six fractured blocks (each of same density), B1 to B6 in order of increasing chips' thickness. The isotropic background of this layer has a P-wave velocity of $2573 \mathrm{~m} / \mathrm{s}$ and a density of $1.175 \mathrm{~g} / \mathrm{cm}^{3}$. The numbers shown indicate model dimensions in millimetres and the model is scaled up by 1:10,000 for spatial dimensions and time measurements.

TABLE 1: Parameters of the fracture models. The dimensions are scaled up to $1: 10000$. Dimensions shown are not converted to real scaling.

\begin{tabular}{lcccccc}
\hline Model number/block & B1 & B2 & B3 & B4 & B5 & B6 \\
\hline Number of layers & 30 & 30 & 30 & 360 & 30 & 360 \\
Number of chips per layer & 360 & 360 & 10800 & 10800 & 10800 & 10800 \\
Total number of chips in model & 10800 & 10800 & 0.20 & 0.25 & 0.30 & 0.35 \\
Chip thickness (mm) & 0.10 & 0.15 & 1.63 & 1.61 & 1.63 \\
Layer thickness (mm) & 1.59 & 1.63 & 1.5 & 1.5 & 1.5 & 1.58 \\
Radius of each chip (mm) & 1.5 & 1.5 & 458640 & 458640 & 458640 & 458640 \\
Volume of base material & 458640 & 458640 & 7.95 & 7.95 & 7.95 & 7.95 \\
Fracture density (\%) & 7.95 & 7.95 & &
\end{tabular}

a fixed diameter of $3 \mathrm{~mm}$ and P-wave velocity of $1300 \mathrm{~m} / \mathrm{s}$ are randomly embedded in the isotropic background of the second layer to make the layer anisotropic. The chips are very thin with very low $S$-wave velocity and therefore act in such a way as to provide weakness to the background material. They are arranged to form six fractured blocks with their thicknesses as $0.10,0.15,0.20,0.25,0.30$, and $0.35 \mathrm{~mm}$, respectively, in each block. Each block is made up of 30 layers of epoxy resin. Once a layer is laid, 360 thin chips are randomly embedded into the layer and another layer is added on the top. The whole procedure is repeated until a total of 30 layers were achieved. All the six fracture blocks have the same fracture density of $7.95 \%$. The fracture density "FD" in this case is defined mathematically as

$$
\mathrm{FD}=\frac{n a^{3}}{V},
$$

where $n$ is the number of chips in the base material, $a$ is the radius of each chip, and $V$ is the volume of the base material. The fracture density for each of the fractured blocks was computed from (1). The vacuum mixing technique was utilized to create the models and the mixing was done at a very fast rate in order to control the homogeneity of the mixture. Details of the fracture model parameters are given in Table 1. The model is constructed with a scale of $1: 10,000$ for spatial dimensions and time measurements. This implies that the model dimensions are multiplied by a factor of 10 in order to get the corresponding field dimensions in metres. The P- and S-wave velocities of the materials in the model were measured by using the pulse and transmission method as in Wei et al. [9]. Measurements were made at an ultrasonic frequency of $250 \mathrm{kHz}$, corresponding to $25 \mathrm{~Hz}$ after appropriate scaling. The model is a simplified analogous representation of a fractured reservoir with varying fracture apertures. Although the simulated fractures may not be real fracture analogues in a typical fractured reservoir setting, this study is expected to provide useful information on the effects 
of voids in the rock on P-wave attenuation and a basis for further theoretical development since there is no theory at the moment to explain this kind of scenario.

\section{Experimental Setup and Data Acquisition}

The experimental setup for the data acquisition is shown in Figure 3. The physical model was submerged in a water tank for ease of data acquisition and coupling of the various parts of the model. 2D reflection data were acquired in a direction perpendicular to the fracture strikes using the pulse and transmission method. The water depth to the top of the model is $100 \mathrm{~mm}$, making the net thickness of the overburden above the fractured layer to be $138 \mathrm{~mm}$. The physical modelling system consists of an ultrasonic pulse source and a receiver system, an analogue/digital converter, and a motor driven positioning system with a precision of $0.1 \mathrm{~mm}$ (Figure 4). The pulse source (transducer) has a size of $20 \mathrm{~mm}$ and produces plane waves in this experiment. The dominant wavelength of the P-wave generated is $11.2 \mathrm{~mm}$ compared with the fracture diameter of $3 \mathrm{~mm}$. The conditon of equivalence of a fractured medium to an anisotropic medium under the long wavelength approximation of the equivalent medium theory is as follows [12]:

seismic wavelength $\gg$ fracture spacing $\gg$ fracture opening

In our seismic physical modelling case, the average fracture spacing is $1.61 \mathrm{~mm}$ while the highest fracture thickness or aperture is $0.35 \mathrm{~mm}$. This implies that $11.2 \mathrm{~mm} \gg 1.61 \mathrm{~mm}$ $\gg 0.35 \mathrm{~mm}$. Thus, the model satisfies the long wavelength approximation and the simulated fractures are expected to cause scattering of seismic wave energy resulting in attenuation. The source and receiver were located on the water surface. For the first shot gather located $500 \mathrm{~mm}$ from the edge of the tank (Figure 3), a single shot was fired into a single receiver at a minimum offset of $16 \mathrm{~mm}$, the receiver was then moved a distance of $2 \mathrm{~mm}$ away, and another shot was fired until a total of 120 receiver positions were occupied at a spacing of $2 \mathrm{~mm}$ for the shot position. The shot was then moved a distance of $2 \mathrm{~mm}$ in the direction of the receiver and the entire procedure repeated. A total of 220 shots were made at $2 \mathrm{~mm}$ intervals. The ultrasonic pulse source has a centre frequency of $250 \mathrm{kHz}$ and a bandwidth of $100-$ $400 \mathrm{kHz}$. The frequency was scaled down by 10,000:1 to fit the scale model dimensions and velocity appropriately. The laboratory model was up-scaled using the geometry scaling. The model dimensions were multiplied by 10 to get their equivalent field scaling in metres while the frequencies were divided by 10 to get their field equivalent in Hertz. Thus, on appropriate scaling, the centre frequency of the pulse source is $25 \mathrm{~Hz}$ and the corresponding bandwidth is 10$40 \mathrm{~Hz}$. Details of the acquisition geometry are summarized in Table 2. Aperture diffraction effect caused by the finite dimension of the transducer aperture might influence the accuracy of attenuation measurement if not corrected for (e.g., [13-16]). This extrinsic effect is mostly prominent when the aperture size is roughly similar to the wavelength. In our physical modelling experiment, the size of the transducer
TABLE 2: Summary of data acquisition geometry-all the acquisition parameters given are not converted to actual scales. The model dimensions and acquisition parameters are scaled up by $1: 10000$ while the frequency is scaled down by $10000: 1$.

\begin{tabular}{lc}
\hline Water depth to base model $(\mathrm{mm})$ & 100 \\
Number of shots & 220 \\
Shot interval $(\mathrm{mm})$ & 2 \\
Receiver interval $(\mathrm{mm})$ & 2 \\
Number of receivers & 120 \\
Minimum offset $(\mathrm{mm})$ & 16 \\
Maximum offset $(\mathrm{mm})$ & 254 \\
Fold of cover & 60 \\
Number of samples & 4096 \\
Sample rate $(\mu \mathrm{s})$ & 0.1 \\
\hline
\end{tabular}

aperture is more than the wavelength of the generated Pwaves and, thus, we assume that aperture diffraction effects are negligible.

\section{Data Processing}

The raw data is made up of 230 shot gathers with 120 traces in each gather. On appropriate scaling to effective field dimensions, the corresponding trace spacing is $20 \mathrm{~m}$, while the minimum and maximum offsets are $160 \mathrm{~m}$ and $2540 \mathrm{~m}$, respectively. Since the data has high signal-to-noise ratio, minimal processing was done on the data in order to preserve all the amplitude information needed for attenuation analysis. Thus, the following processing sequences were applied: geometry configuration, common midpoint (CMP) sorting, trace-muting, velocity analysis, and NMO correction. Stacking was also included in the processing sequence for ease of event identification and picking of the travel times to the target layers, even though the $Q$ values were estimated from the prestack CMP gathers. A sample CMP NMO-corrected gather is shown in Figure 5. The reflections that we analysed are those from the top of the base model (blue arrow), the top of the fractured layer (red arrow), and the bottom of the fractured layer (green arrow), with the geometry shown in Figure 2. We used an offset range of 160$940 \mathrm{~m}$ for all the CMP gathers analysed and all the target reflections are continuous for this offset range, respectively.

\section{Attenuation Measurement}

Among the various methods of measuring attenuation from seismic data, the spectral ratio method is the most common method perhaps because it is easier to use and more stable (e.g., [17-20]). In this paper, we used the QVO method (introduced by Dasgupta and Clark [20]), which is an extension of the spectral ratio method to estimate the induced attenuation (inverse of the seismic quality factor $Q$ ) in the fractured layer. Our physical modelling data have high $S / N$ ratio and as such we assumed that interference effects caused by multiples or any other noise interference are either absent or too small to cause any significant bias on the $Q$ estimates. 


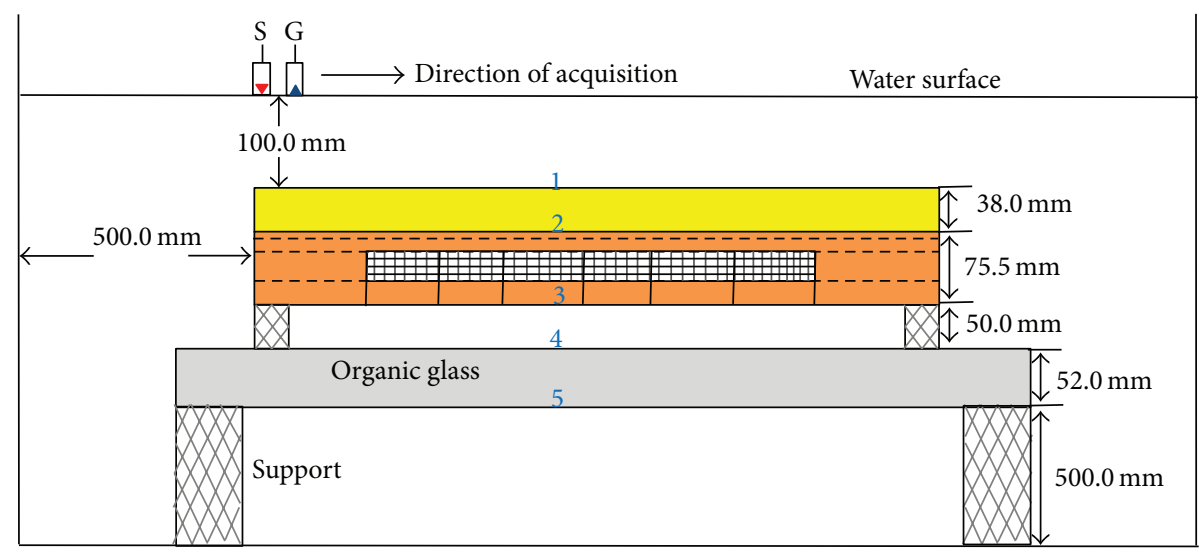

Figure 3: Experimental setup for data acquisition. The base model is submerged in a water tank. S and $G$ are the shot and receiver positions, respectively. The model dimensions shown are scaled up by $1: 10000.2 \mathrm{D}$ reflection data were acquired in a direction normal to the fracture strikes. The blue numbers indicate the reflection interfaces.

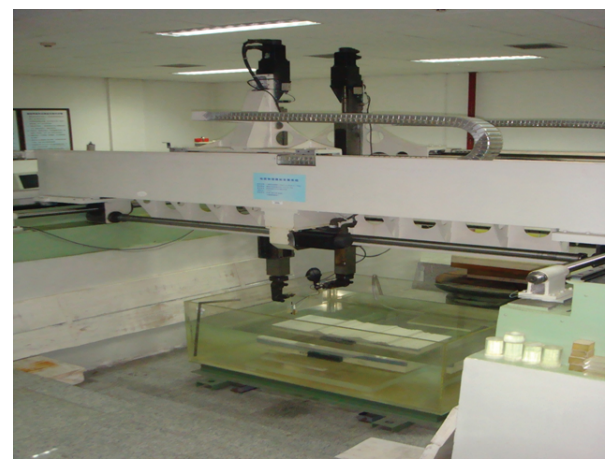

FIGURE 4: The physical modelling system for 2D data acquisition in the laboratory. The base model is submerged in a water tank while the source and receiver were moved along the water surface.

As a reference, we used the first trace from the top model reflection at an offset of $160 \mathrm{~m}$ (which is minimum offset in the data) in each preprocessed CMP gather, for comparison of the spectral ratios and a constant window length of $160 \mathrm{~ms}$. We then computed the spectral amplitude ratios according to (2) and performed a least-squares regression of the logarithm of the power spectral ratio (LPSR) against frequency:

$$
\ln \frac{A_{2}^{2}}{A_{1}^{2}}=\ln \frac{P_{2}}{P_{1}}=2 \ln (R G)-\frac{2 \pi f}{Q}\left(t_{2}-t_{1}\right),
$$

where $f$ is frequency, $R$ is the reflectivity term, $G$ is the geometrical spreading factor, $A_{2}$ is the spectral amplitude of the target reflection (top or bottom of fractured layer), and $A_{1}$ is the spectral amplitude of the reference trace while $P_{1}$ and $P_{2}$ are the respective spectral powers (square of amplitudes), $t_{1}$ and $t_{2}$ are the corresponding travel times, and $Q$ is the seismic quality factor down to the reflector. The slope of the regression " $p$ " is given by

$$
p=-\frac{2 \pi\left(t_{2}-t_{1}\right)}{Q} .
$$

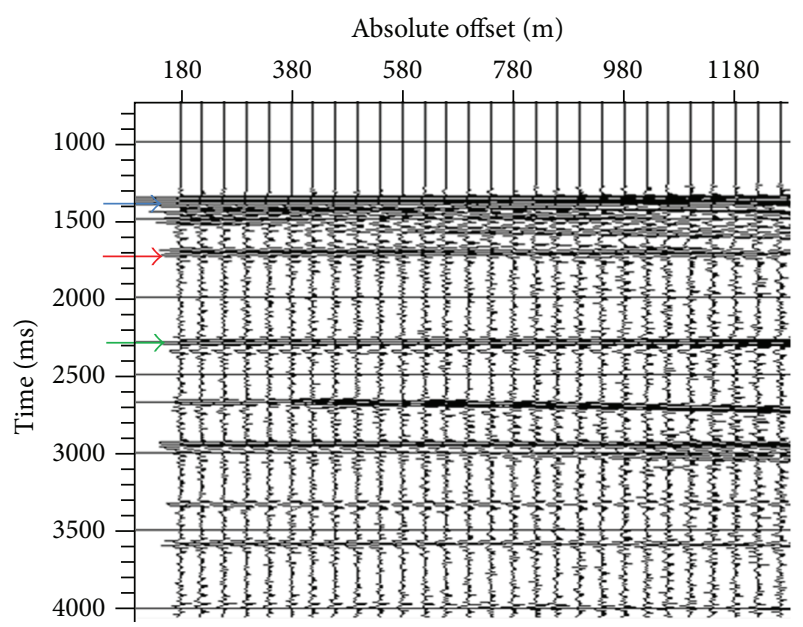

FIGURE 5: Sample NMO-corrected gather. The blue, red, and green arrows indicate the reflections from the top of the base model and the top and bottom of the fractured layer, respectively.

Sample plots of the logarithm of the power spectral ratios versus frequency are shown in Figure 6 and are approximately linear in the frequency bandwidth of $10-40 \mathrm{~Hz}$. This linearity is an approximation and the frequency bandwidth lies within the signal bandwidth of the pulse source $(10-40 \mathrm{~Hz})$. This bandwidth was kept constant for all the trace pairs analyzed. There is no signal below the frequency of $10 \mathrm{~Hz}$ and hence the scatter in the spectral plots (Figure 6). The same is applicable to frequencies beyond $40 \mathrm{~Hz}$.

The best fitting slope $p$, defined in (3), between these limits was then obtained by least-squares regression. Following (3), this slope in the absence of a zero-offset reference trace in the data can be written as

$$
p=\frac{2 \pi}{Q}\left(t_{o, \text { ref }}-t_{o}+\frac{x^{2}}{2}\left\{\frac{1}{t_{o, \text { ref }} v^{2}{ }_{\text {ref, } \mathrm{rms}}}-\frac{1}{t_{o} v^{2}{ }_{\mathrm{rms}}}\right\}\right),
$$

where $x$ is offset, $t_{o \text {,ref }}$ is the zero-offset travel-time of reference trace, $t_{o}$ is the zero-offset travel-time of target reflection, 


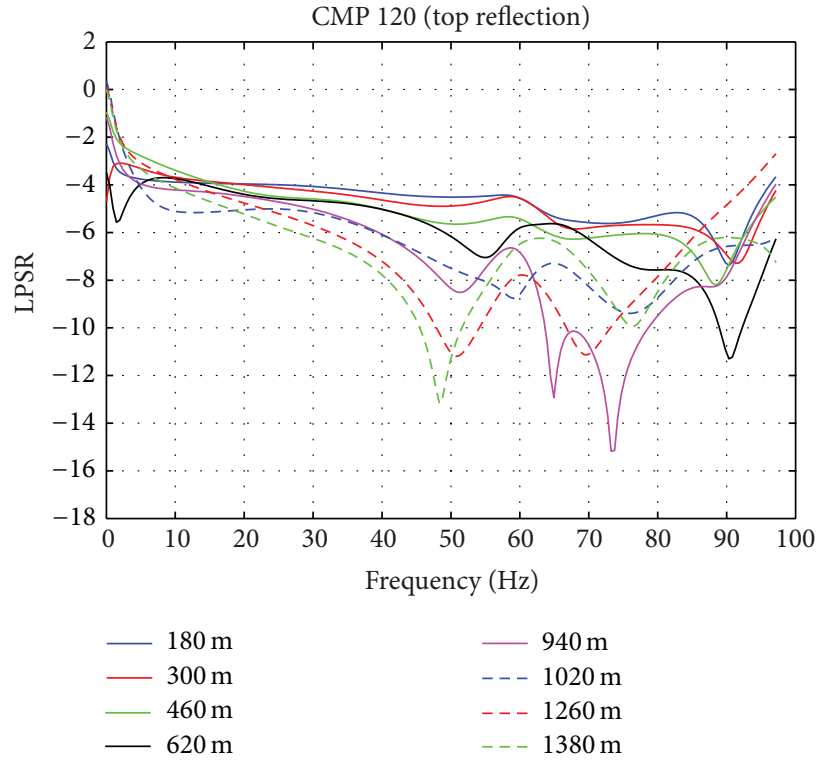

(a)

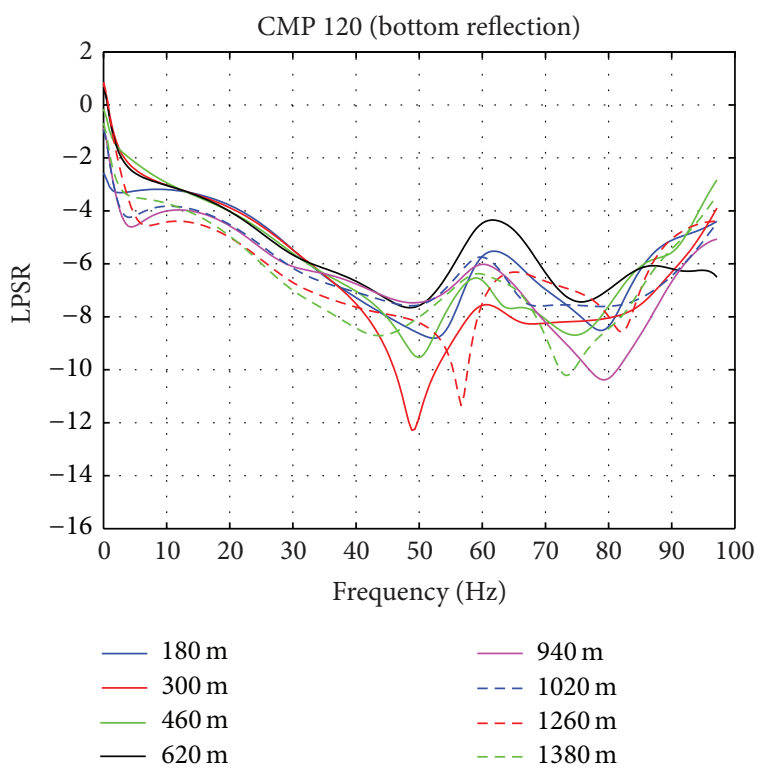

(b)

FIGURE 6: Log spectral power ratio (LSPR) against frequency plots (CMP 120). (a) Top fractured-layer reflection. (b) Bottom fractured-layer reflection. Plots are approximately linear within frequency range of $10-40 \mathrm{~Hz}$.

and $V_{\text {ref,rms }}$ and $V_{\text {rms }}$ are the root mean square velocities of the reference trace and target reflection, respectively. Equation (4) indicates a linear relationship between the spectral ratio slope and the square of offsets. Thus, we carried out another least-squares regression of the spectral ratio slopes against the square of the offsets to get the zero-offset slope (LSPR slope intercept, $I$ ) given by

$$
I=\frac{2 \pi\left(t_{o, \text { ref }}-t_{o, 2}\right)}{Q}
$$

The seismic quality factor $Q$ down to the top of the fractured layer was then computed from (5). The entire procedure was repeated for the bottom fractured-layer reflection and all the CMP gathers analyzed. With the pair of $Q$ values computed down to the top and bottom of the fractured-layer in each CMP gather, we finally estimated the interval seismic quality factor, $Q_{i}$ in the fractured-layer using the equation [20]:

$$
Q_{i}=\frac{\left[t_{o, 2}-t_{o, 1}\right]}{t_{o, 2} / Q_{2}-t_{o, 1} / Q_{1}},
$$

where $Q_{1}$ and $Q_{2}$ are the seismic quality factors down to top and bottom of the fractured-layer respectively while $t_{o, 1}$ and $t_{o, 2}$ are the corresponding zero-offset travel times. The zero-offset travel times were obtained by extrapolation on the time axis since the minimum offset in the data is $160 \mathrm{~m}$ after appropriate scaling. Sample plots of the slopes against the square of the offsets are shown in Figure 7. The red dashed lines indicate a $95 \%$ confidence interval on the best fitting (green) line in each case.

\section{Results}

The results of the attenuation analysis show that the magnitude of the logarithm of the spectral ratio slopes varies with the thickness of the chips, indicative of a systematic dependence of the scattering attenuation on the thickness (which is meant to model the effect of aperture in an underground crack). The absolute value of the slope increases with the chip's thickness which indicates an increase in scattering and hence attenuation. Higher interval $Q$ values (low attenuation) are obtained for the CMP(s) at both ends of the survey line (below CMP 100 and beyond CMP 400) where there are no fractures (Figure 8). The $Q$ values decrease systematically in the direction of increasing chips' thickness from the left edge of block B1 (CMP 120) to the right edge of block B6 (CMP 410), implying more scattering in the direction of increasing chips' thickness. This trend is shown in Figure 9 for the $\mathrm{CMP}(\mathrm{s})$ corresponding to the centres of the fractured blocks, respectively.

\section{Discussion}

A set of aligned fractures is known to greatly influence the propagation of seismic waves by causing scattering of the wave energy resulting in seismic coda trailing the primary reflections. Such scattering effects have been shown to be useful in providing information about the fracture properties (e.g., [21-23]). The resultant effect of the scattering is a gradual loss in the wave energy which can be observed in amplitude changes as the wave propagates through the medium. This effect can be produced by a set of fractures at different scale length and the popular Hudson theory $[6,7]$ fails to account for these scale length issues. The results of 


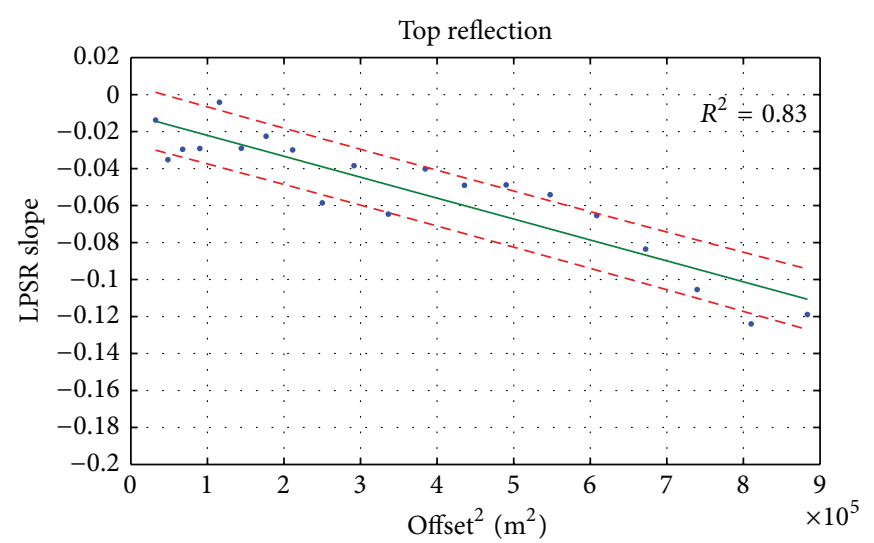

(a)

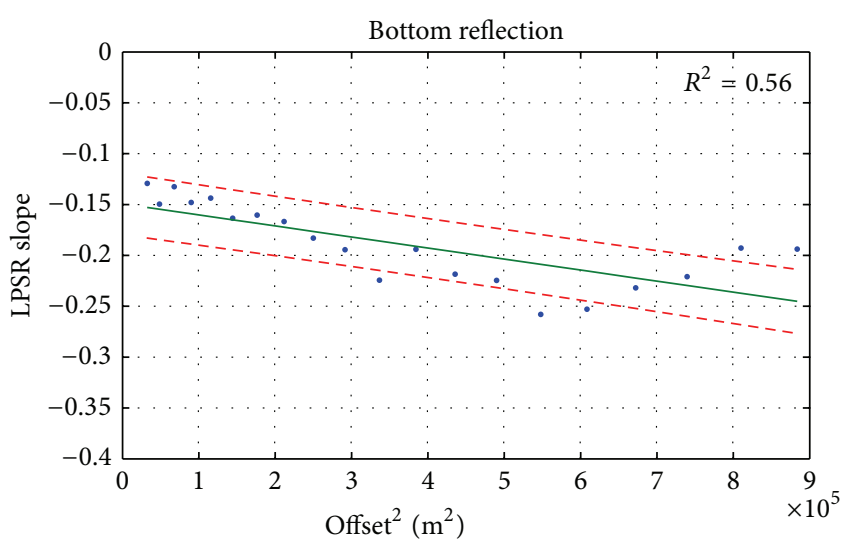

(b)

FIGURE 7: Least-squares regression of logarithm of power ratio slopes against the square of offset (CMP 260). The asterisks indicate the data point; the green line indicates the fitted line while the red dashed lines indicate a $95 \%$ confidence interval on the fitted line.

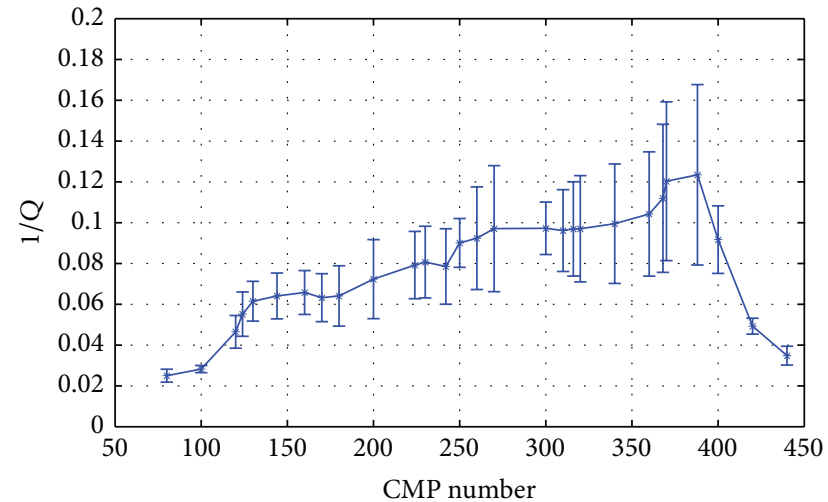

FIGURE 8: 1/Q results for the fractured layer against CMP numbers: fractured blocks lie between CMP(s) 120 and 410, respectively. There is a systematic increase in the induced attenuation (inverse $Q$ ) in the direction of increasing chips' thickness from CMP 120 to CMP 410. However, the attenuation is less at the ends of the line where there are no fractures.

the seismic physical modelling studies of Wei et al. $[9,10]$ demonstrate that a set of aligned fractures with different diameters but same thickness and fracture density significantly affect both $\mathrm{P}$ - and S-wave velocities especially for wave propagation parallel to the fracture strike. The wave velocity increases with diameter as a result of reduced scattering. Further seismic physical modelling studies by Wei et al. [9] also demonstrate that a set of aligned fractures with the same density and diameter but varying thickness or aperture has a strong influence on the P-wave amplitude and waveforms for transmitted P-wave data. The waveform is highly attenuated with increasing thickness or aperture especially for wave propagation perpendicular to the fractures.

In this paper, we have quantified the scattering effects caused by a set of fracture models with the same density and diameter but varying thicknesses or apertures for P-wave reflection data through attenuation estimates. The fracture

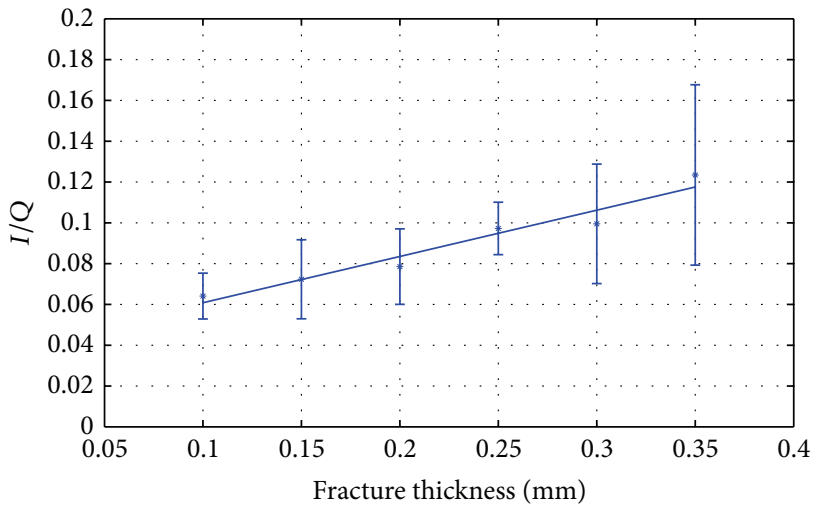

FIGURE 9: I/Q profile against chip's thickness. $Q$ decreases with increasing chip's thickness, implying more attenuation.

models are simulated by embedding thin penny-shaped chips into an isotropic background medium. These chips act in such a way as to cause a weakness in the medium, and thus we exploit this weakness to simulate fractures. The two layers in the base model were glued together to ensure good coupling between them. A rough estimate of the transmission coefficient at the top of the fractured layer gives a transmission coefficient $>90 \%$ which is also indicative of a highquality coupling between the two layers of the model. Data acquisition in a water tank where the model was submerged also facilitates a good coupling of the the source and receivers as well as the other components of the model. The results of our analysis show that P-wave attenuation has a direct relationship with fracture thickness or aperture. Attenuation (inverse $Q$ ) increases systematically and linearly with fracture thickness, implying proportionately more scattering of the wave energy as the wave propagates in the direction of increasing thickness or aperture. Although the simulated fractures may not be real fracture analogues in a typical fractured reservoir setting, the results provide information which might be useful in examining the effects of voids in the rock on $\mathrm{P}$-wave attenuation and may provide a basis 
for further theoretical development to distinguish the effects caused by thin microcracks and large open fractures.

\section{Conclusions}

We demonstrate that a set of aligned fractures causes scattering of seismic wave energy, resulting in attenuation. A direct relationship exists between attenuation and fracture thickness or aperture, indicating the potential of using Pwave attenuation to distinguish the effect caused by thin microcracks from that caused by large open fractures. Similar studies by Wei et al. [9] on P-wave transmitted data have shown that a set of aligned fractures with the same density and diameter but different apertures or thickness cause significant attenuation in the waveform amplitude especially for wave propagation normal to the fracture strike direction. Our findings show consistency with these observations and thus provide a physical basis of using P-wave attenuation attribute to distinguish the effects caused by thin microcracks and large open fractures from seismic data.

\section{Conflict of Interests}

The authors declare that there is no conflict of interests regarding the publication of this paper.

\section{Acknowledgments}

The authors are grateful to the China National Petroleum Corporation (CNPC) for providing the funds to conduct the physical modelling experiments under a collaboration agreement between the CNPC Geophysical Key Laboratory at the China University of Petroleum and the Edinburgh Anisotropy Project (EAP) at the British Geological Survey (BGS) and the permission to publish the results. Thanks are due to Professor Wang and Professor Di both of the China University of Petroleum for being good hosts during the experimental period. This work was also partially supported by the National Natural Science Foundation of China. Special thanks are due to the Akwa Ibom State University (AKSU), Nigeria, for sponsoring Ekanem's Ph.D. studies at the University of Edinburgh.

\section{References}

[1] M. Schoenberg and C. M. Sayers, "Seismic anisotropy of fractured rock," Geophysics, vol. 60, no. 1, pp. 204-211, 1995.

[2] S. A. Hall, J. M. Kendall, O. I. Barkved, and M. C. Mueller, "Fracture characterization using P-wave AVOA in 3D OBS data," SEG expanded abstract, pp. 1409-1412, 2000.

[3] D. Gray and K. Head, "Fracture detection in Manderson Field: a 3-D AVAZ case history," Leading Edge, vol. 19, no. 11, pp. 1214$1221,2000$.

[4] X.-Y. Li, Y.-J. Liu, E. Liu, F. Shen, L. Qi, and Q. Shouli, "Fracture detection using land 3D seismic data from the Yellow River Delta, China," Leading Edge, vol. 22, no. 7, pp. 680-684, 2003.

[5] M. Luo and B. J. Evans, "An amplitude-based multiazimuthal approach to mapping fractures using P-wave 3D seismic data," Geophysics, vol. 69, no. 3, pp. 690-698, 2004.
[6] J. A. Hudson, "Overall properties of a cracked solid," Mathematical Proceedings of the Cambridge Philosophical Society, vol. 88, no. 2, pp. 371-384, 1980.

[7] J. A. Hudson, "Wave speeds and attenuation of elastic waves in material containing cracks," Geophysical Journal, vol. 64, no. 1, pp. 133-150, 1981.

[8] R. Zimmermann and I. Main, "Hydromechanical behaviour of fractured rocks," in Mechanics of Fluid-Saturated Rocks, Y. Gueguen and M. Bouteca, Eds., pp. 361-419, Academic Press, London, UK, 2003.

[9] J. Wei, B. Di, and X.-Y. Li, "Effect of fracture scale length and aperture on seismic wave propagation: an experimental study," Journal of Seismic Exploration, vol. 16, no. 2-4, pp. 265-280, 2007.

[10] J. Wei, B. Di, and Q. Wang, "Experimental study on the effect of fracture scale on seismic wave characteristics," Petroleum Science, vol. 5, no. 2, pp. 119-125, 2008.

[11] M. Chapman, "Frequency-dependent anisotropy due to mesoscale fractures in the presence of equant porosity," Geophysical Prospecting, vol. 51, no. 5, pp. 369-379, 2003.

[12] A. Bakulin, V. Grechka, and I. Tsvankin, "Estimation of fracture parameters from reflection seismic data-Part I: HTI model due to a single fracture set," Geophysics, vol. 65, no. 6, pp. 1788-1802, 2000.

[13] H. Seki, A. Granato, and R. Truell, "Diffraction effects in the ultrasonic field of a piston source and their importance in the accurate measurement of attenuation," Journal of the Acoustical Society of America, vol. 28, pp. 230-238, 1956.

[14] E. P. Papadakis, "Ultrasonic diffraction loss and phase change in anisotropic materials," Journal of the Acoustical Society of America, vol. 40, pp. 863-876, 1966.

[15] M. B. Gitis and A. S. Khimunin, "Diffraction corrections for measurements of the absorption coefficient and velocity of sound," Soviet Physics, vol. 14, pp. 305-310, 1969.

[16] E. P. Papadakis, K. A. Fowler, and L. C. Lynnworth, "Ultrasonic attenuation by spectrum analysis of pulses in buffer rods: method and diffraction corrections," Journal of the Acoustical Society of America, vol. 53, no. 5, pp. 1336-1343, 1973.

[17] P. S. Hauge, "Measurements of attenuation for vertical seismic profiles," Geophysics, vol. 46, no. 11, pp. 1548-1558, 1981.

[18] J. Pujol and S. Smithson, "Seismic wave attenuation in volcanic rocks from VSP experiments," Geophysics, vol. 56, no. 9, pp. 1441-1455, 1991.

[19] R. Tonn, "The determination of the seismic quality factor Q from VSP data: a comparison of different computational methods," Geophysical Prospecting, vol. 39, no. 1, pp. 1-27, 1991.

[20] R. Dasgupta and R. A. Clark, "Estimation of Q from surface seismic reflection data," Geophysics, vol. 63, no. 6, pp. 2120-2128, 1998.

[21] C. A. Schultz and M. N. Toksoz, "Reflections from a randomly grooved interface: ultrasonic modelling and finite-difference calculation," Geophysical Prospecting, vol. 43, no. 5, pp. 581-594, 1995.

[22] M. E. Willis, D. R. Burns, R. Rao, B. Minsley, M. N. Toksöz, and L. Vetri, "Spatial orientation and distribution of reservoir fractures from scattered seismic energy," Geophysics, vol. 71, no. 5, pp. O43-O51, 2006.

[23] D. R. Burns, M. E. Willis, M. N. Toksöz, and L. Vetri, "Fracture properties from seismic scattering," Leading Edge, vol. 26, no. 9, pp. 1186-1196, 2007. 

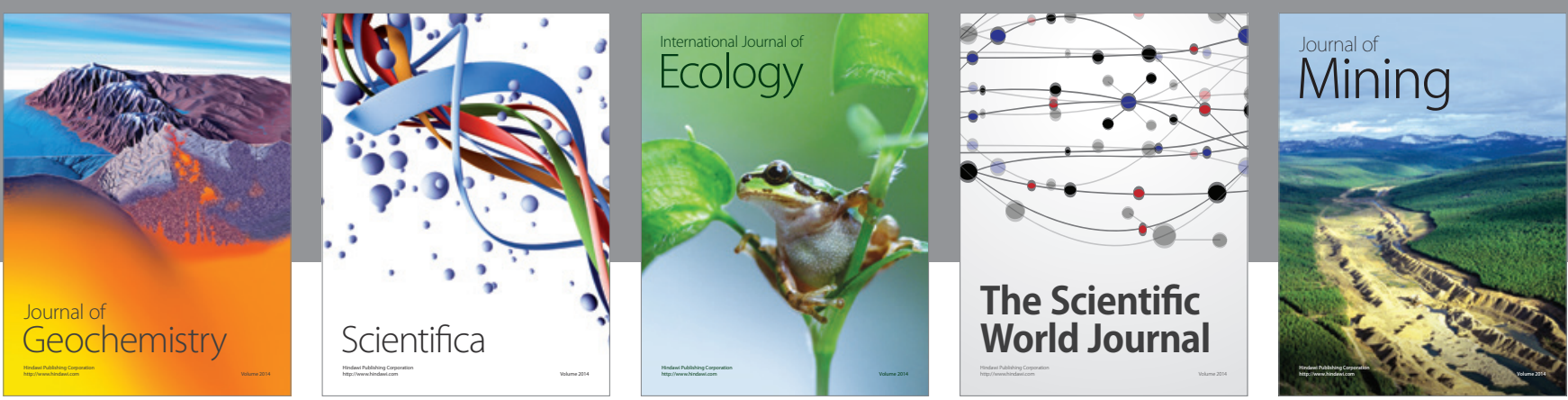

The Scientific World Journal
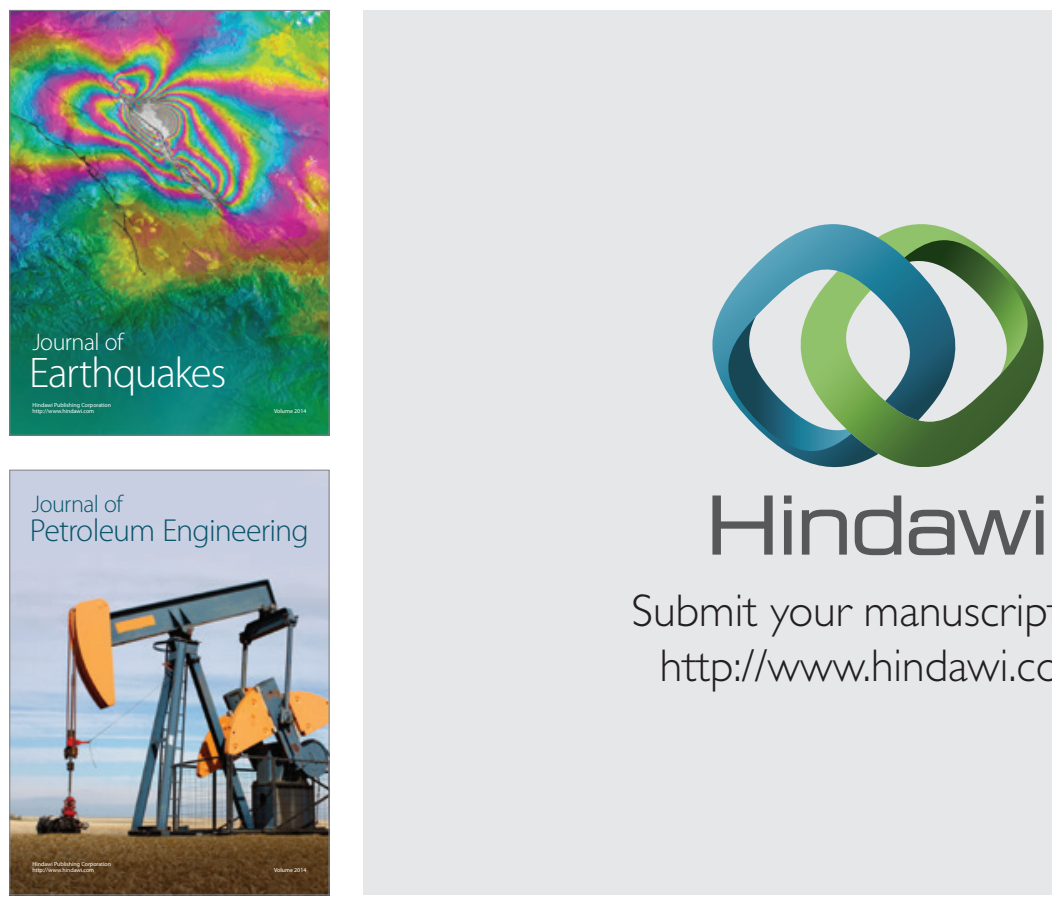

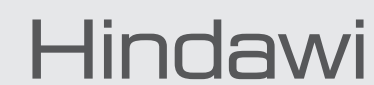

Submit your manuscripts at

http://www.hindawi.com
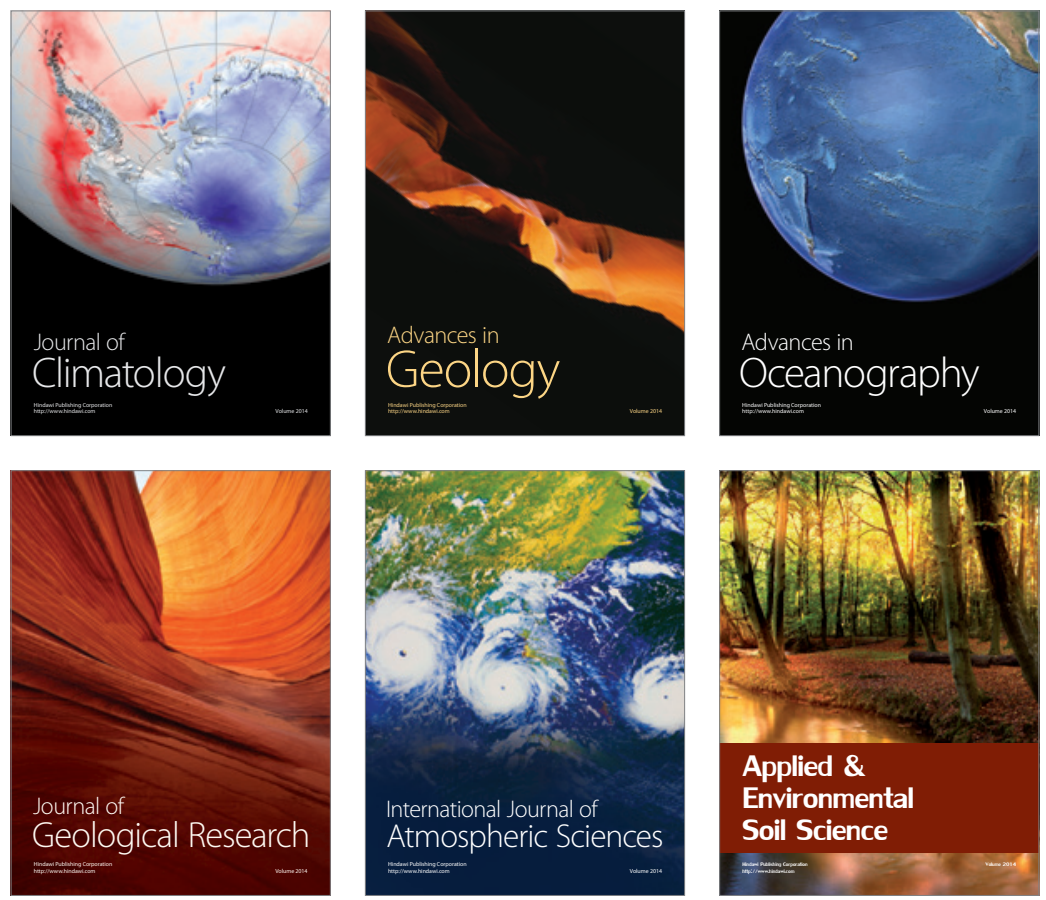
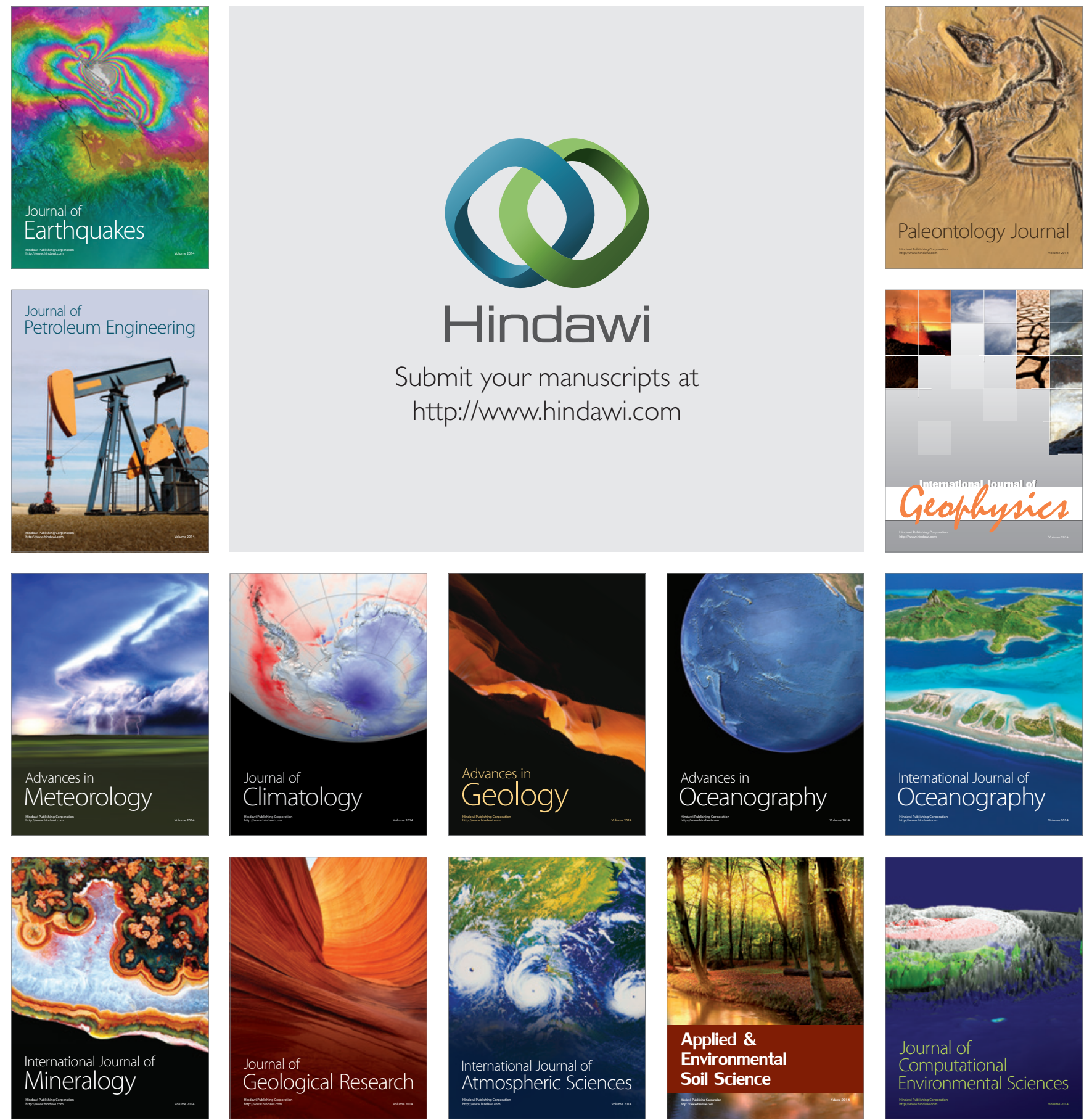